\title{
Human breast tissue characterisation with small-angle X-ray scattering
}

\author{
M. Fernández ${ }^{\text {a,e,* }}$ J. Keyriläinen ${ }^{\text {a,b }}$, M.-L. Karjalainen-Lindsberg ${ }^{\text {c }}$, M. Leidenius ${ }^{\text {d, }}$ \\ K. von Smitten ${ }^{\mathrm{d}}$, S. Fiedler ${ }^{\mathrm{e}}$ and P. Suortti ${ }^{\mathrm{a}}$ \\ ${ }^{a}$ Department of Physical Sciences, POB 64, FIN-00014 University of Helsinki, Finland \\ ${ }^{\mathrm{b}}$ Department of Physics, HUCH Cancer Center, Helsinki University Central Hospital, РOB 180, \\ FIN-00029 HUS, Helsinki, Finland \\ ${ }^{\mathrm{c}}$ Department of Pathology, HUCH Laboratory Diagnostics, Helsinki University Central Hospital, \\ POB 400, FIN-00029 HUS, Helsinki, Finland \\ ${ }^{\mathrm{d}}$ Department of Surgery, Maria Hospital, Helsinki University Central Hospital, POB 580, \\ FIN-00029 HUS, Helsinki, Finland \\ ${ }^{\mathrm{e}}$ European Synchrotron Radiation Facility, BP 220, F-38043 Grenoble, France
}

\begin{abstract}
Small-angle X-ray scattering (SAXS) patterns from breast tissue samples are compared with their histology. Formalin fixed human breast tissue specimens containing ductal and lobular carcinoma were studied. Histo-pathological information is compared with the scattering data, and there is a clear spatial correlation. Supra-molecular organisation of collagen fibrils is modelled and the model is used to create scattering maps. The model parameters include the axial periodicity ( $d$-spacing), radius and packing of the fibrils, and these are derived from comparison with the experimental scattering patterns. The $d$-spacing is to $0.5 \%$ larger in malignant zones of the tumours than in the healthy zones. There are also characteristic differences in the fibril diameter and packing.
\end{abstract}

\section{Introduction}

Breast cancer is one of the principal causes of death among women in developed countries [5]. The mortality of the disease is considerably reduced, if tumours are found at an early stage of growth [9]. Frequently the disease may be fully symptomless, and therefore mammographic screening is carried out among women over 50 years old in many countries. Nevertheless, in young dense breast, the cases of false positives and/or missed tumours, false negatives, are frequent [12], especially in the case of the lobular carcinoma, and/or in the absence of micro-calcifications or masses of different densities.

Mammography is based on the absorption of X-rays in the tissues, and it reveals changes in density and morphology. The absorption contrast is weak, and the mammographic signs of cancer are subtle, and new methods for imaging of breast cancer are being developed [13]. In particular, the contrast in soft tissues such as mammary gland is much more enhanced with phase contrast methods.

The nature of the breast tumours is indicated either by morphology (in the core needle biopsies) or radiographic signs (in the mammograms). In the case of malignant diagnosis an increasingly employed

\footnotetext{
${ }^{*}$ Corresponding author: Manuel Fernández, Department of Physical Sciences, POB 64, FIN-00014 University of Helsinki, Finland. E-mail: Manuel.Fernandez@Helsinki.FI.
} 
treatment is conserving surgery in combination with chemotherapy, hormone treatment or postoperative radiotherapy. However, before proceeding to definitive treatment, the confirmation of diagnosis is always done by the histological biopsy. In a core needle biopsy, the needle is introduced into the tissue and a little piece of the tumour is extracted. Then the biopsy is examined by histo-pathological methods in order to determine the nature of the tumour.

Breast tumour growth (hyperplasia) is closely related to collagenosis, and connective tissue may wrap the tumour and encapsulate it in the healthy tissue. Fibrillar collagen is newly formed in the tumours, and it has a supra-molecular structure different from the one found in healthy tissues $[10,11]$. Smallangle X-ray scattering (SAXS) is an efficient technique for retrieving the supra-molecular structure of the breast tissues, especially those rich in collagen [4]. Different "signatures" of the SAXS patterns of the tissues can be systematically studied and the scattering signals can be mapped and compared with histo-pathology of the samples.

\section{Small-angle X-ray scattering}

SAXS is a powerful method to determine structural features of molecular systems in soft tissue and their different degrees of organisation. The scattering vector $\boldsymbol{k}$ is defined by two unit vectors that indicate the incident $\left(s_{0}\right)$ and the scattered beam $(s)$, respectively (Fig. 1),

$$
\boldsymbol{k}=(2 \pi / \lambda)\left(s-s_{0}\right)
$$

The modulus of the scattering vector is

$$
k=(4 \pi / \lambda) \sin \theta,
$$

or alternatively,

$$
s=2 \sin \theta / \lambda=k / 2 \pi
$$

Here $\theta$ is half of the scattering angle. Both $k$ and $s$ will be used in the following.

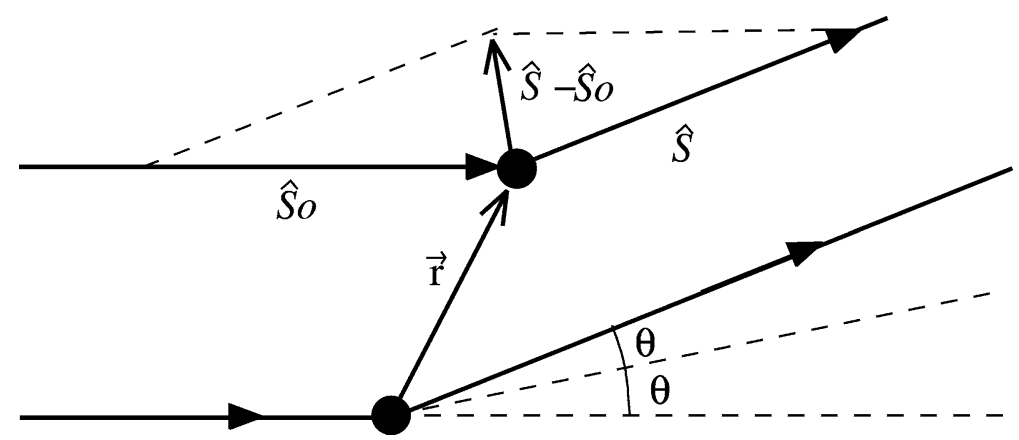

Fig. 1. Geometry of elastic scattering of X-rays indicated by unit vectors $s_{\mathrm{o}}$ and $s$. The scattering vector $k=(2 \pi / \lambda)\left(s-s_{\mathrm{o}}\right)$ and the scattering angle is $2 \theta$. 
The Bragg Law gives the condition for constructive interference of scattering from a structure that has a periodicity $d$ in the direction of the scattering vector,

$$
n \lambda=2 d \sin \theta
$$

where $n=1,2,3, \ldots$ are the different orders. Combining Eqs (3) and (4),

$$
d=n / s
$$

This equation is particularly useful, because it gives the real space periodicity $d$ in terms of the positions of the diffraction maxima, $s$.

The scattering amplitude in the units of the electron scattering length (electron classical radius) is the so-called structure factor,

$$
F(s)=\int \rho(\boldsymbol{r}) \exp (\mathrm{i} \boldsymbol{k} \cdot \boldsymbol{r}) \mathrm{d}^{3} \boldsymbol{r},
$$

and $\rho$ is the electron density. Basically, the scattering amplitude is the Fourier transform of the electron density of the scatterer. The observable quantity is the intensity, which is the Fourier transform of the autocorrelation function $P(\boldsymbol{r})$ of the electron density,

$$
I(s)=F(s) F^{*}(s)=\int P(\boldsymbol{r}) \exp (\mathrm{i} \boldsymbol{k} \cdot \boldsymbol{r}) \mathrm{d}^{3} \boldsymbol{r} .
$$

The intensity distribution of X-ray scattering contains information about the structure of the object on many different levels. Periodicity in the atomic scale produces maxima at large values of $s$, while the intensity modulations due to macromolecules and their assemblies are seen at small $s$, i.e., in the SAXS regime. Typical length scales for these objects are from a few nanometers to several hundred nanometers, so that with $0.1 \mathrm{~nm}$ radiation the SAXS pattern of a tissue sample is observed at scattering angles of one degree and less. Specific formulae for SAXS can be given only for isolated, randomly oriented independent objects, but some of the results are valid under quite general conditions [6]. At the limit of forward scattering the intensity is proportional to the square of the Fourier transform of the shape function of the object, i.e., the object size can be deduced from the intensity at very small $k$. Another important general result is the Porod law, which gives the intensity of scattering at the large values of $k$ in the SAXS pattern. For a 3-dimensional object with a smooth surface the asymptotic form of intensity is

$$
I(s)=2 \pi(\Delta \rho)^{2} S / k^{4}
$$

Here $\Delta \rho$ is the density difference between the object and its surroundings, and $S$ is the surface area of the scatterers per unit mass. Similar results are obtained for thin disks and long rods, for which the power law exponents are -2 and -1 , respectively. For the present case, particularly relevant are polymer chains, which are locally rod-like but become coils over large distances. For these the power law exponent is -2 or $-5 / 3$.

Tissues are made of hierarchical molecular and supra-molecular structures, so that the requirement of independent scatterers is not met. However, realistic models can be constructed and the corresponding 
diffraction patterns can be calculated. Model parameters are obtained from comparison with the experimental data, so that the tissues may be characterised by these parameters. In the following, a model is presented for one of the most ordered tissue components, namely fibrillar collagen.

\section{Collagen}

Collagen is a protein, a polypeptide chain of amino acids, where every 3rd residue is Glycine (Gly$\mathrm{Xxx}$-Yyy). Collagen type I and III are found in the connective tissue of breast, and both types are fibrillar [8]. The molecule of fibrillar collagen consists of a triple $\alpha$-helix, coiled up as a rope [7]. Collagen type I has two identical chains, and the third one is different. Collagen III has all three identical $\alpha$-chains. In fibrils, collagen molecules pack laterally to each other with hydrogen bonds, in an approximately hexagonal close-packed (hcp) structure [14]. Longitudinally, collagen molecules bind to each other in a staggered arrangement. This is a periodic structure, repeated along the fibril axis. The fibrils eventually pack together also in a near-hcp arrangement. This is the supra-molecular structure that gives rise to a characteristic SAXS pattern of collagen.

Collagen fibrils in healthy breast tissue have a radius of about $90 \mathrm{~nm}$, have an inter-fibrillar distance of approximately $100 \mathrm{~nm}$, and the axial periodicity ( $d$-spacing) is approximately $65 \mathrm{~nm}$.

\subsection{SAXS from collagen}

Scattering from a single collagen fibril is well known [14], and also scattering from closely packed fibrils [2,3]. For scattering from a single fibril one must consider two cases: well-oriented (referred to the beam direction) and randomly oriented fibrils. The SAXS pattern is obtained as a linear combination of the contributions from the orientation and size distributions of the fibrils. The contribution from welloriented fibrils can be divided into equatorial and meridional directions. In the equatorial direction there is intensity modulation, which can be described by Bessel functions, and these provide estimates for the distribution of the fibril radii. In the meridional direction many orders of distinct Bragg reflections are observed, and these give the axial $d$-spacing. The contribution of the randomly oriented fibrils is that of scattering from long rods, which falls off as $k^{-1}$. The fibrils are closely packed, and the interference gives rise to a broad maximum at small $k$-values in the equatorial direction. In spite of its simplicity, this model gives a good description of the observed SAXS pattern [4].

\subsection{Collagen degradation}

SAXS patterns give information about the supra-molecular arrangement of collagen, so can be used to identify modifications of such structures. Tissues containing collagen can be classified by the fibril diameter, their packing, and by their axial periodicity. It has been demonstrated that collagen fibrils in healthy tissues have molecular characteristics different from those in malignant tumours $[10,11]$. The SAXS patterns from collagen reflect these differences. For instance, the radii of the fibrils and distances between them are larger in the benign case than in the malignant case, while the axial periodicity in the malignant lesions is about $1 \%$ bigger than in healthy tissue [4].

Another important indication that changes take place in the collagen when cancer develops is the change in the average scattered intensity in certain regions of the SAXS pattern. For instance, the background intensity between the 5th and 6th collagen peaks is significantly higher in the case of invaded 
collagen ${ }^{1}$ than in the case of totally healthy collagen from the same tumour. Other degradation indicators are also possible to measure, but those are not so evident. For instance, there is a loss of sharpness of the collagen peaks, which reduce their maximum intensity with respect to the background.

\section{Experiment}

The experiment was carried out at ID02 High Brilliance beamline of the European Synchrotron Radiation Facility (ESRF) in Grenoble, France. The ESRF is a $6 \mathrm{GeV}$ third generation synchrotron radiation source, which offers a wide range of possibilities to X-ray research. In our case, an undulator was used as the source of radiation, which was monochromatized using Bragg reflections from perfect silicon crystals and focused with a toroidal mirror. A two-dimensional position sensitive detector ${ }^{2}$ was used to acquire the scattering patterns. The integration time was typically of 20-50 milliseconds. The set-up is shown schematically in Fig. 2.

The samples were formalin fixed, and they were sealed hermetically in a holder between Kapton ${ }^{3}$ foils. The diameter of the samples was $20 \mathrm{~mm}$, and the thickness $1 \mathrm{~mm}$. The samples were scanned through a $200 \mu \mathrm{m}$ beam in steps of $1 \mathrm{~mm}$ to $0.25 \mathrm{~mm}$.

\subsection{Sample preparation}

The samples used in this work are human breast tumours and tissues from surgically excised specimens. These excised specimens were flash frozen in liquid nitrogen and stored in freezer at $-80^{\circ} \mathrm{C}$, until their use. Cylindrical pieces, containing tumour and non-tumour tissue were cut off from the specimens while frozen. Several transversal slices were cut from each cylinder. Three holes parallel to the cylinder axis were made using a hollow needle. Surgical marking dyes were introduced in the holes, which were used as reference marks. Immediately after, a thin slice was cut off, let defrost and submerged in formalin ${ }^{4}$. As soon as the sample was formalin fixed, it was processed for histological examination ${ }^{5}$ by a pathologist. The histology of this slice was used as guide to select SAXS measurement points during the experiment. The actual SAXS samples were cut off from the cylindrical pieces right next to the one

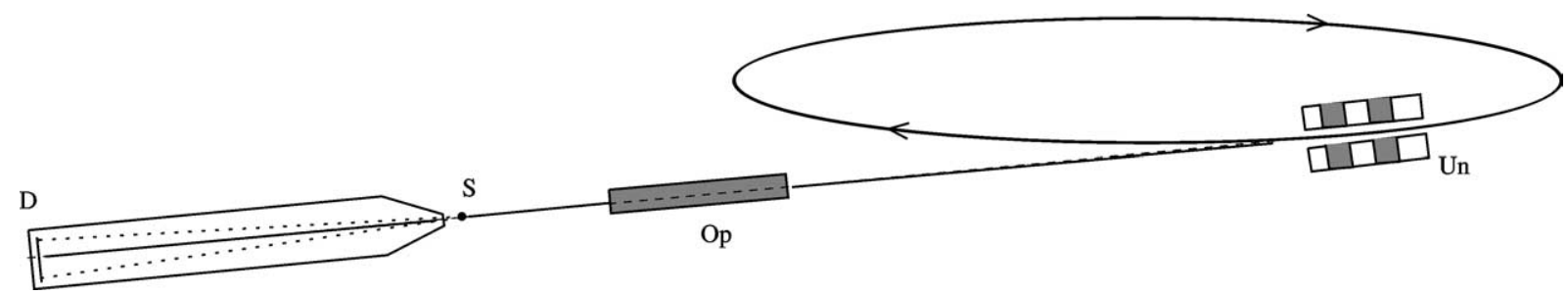

Fig. 2. Schematic presentation of the experiment set-up. An undulator (Un) is used as the X-ray source at ID02 beamline of the ESRF. After several optical components (Op), the beam is monochromatic and focused. The sample position and detector are indicated by $\mathrm{S}$ and $\mathrm{D}$, respectively.

\footnotetext{
${ }^{1}$ Invaded collagen has cancer cells among the fibre strands, which is a consequence of a cancer metastasis or spreading of the cancer cells to surrounding regions.

${ }^{2}$ Detector developed at ESRF: Frelon CCD [1].

${ }^{3}$ Polyamide polymer film (Kapton, DuPont de Nemours, France).

${ }^{4}$ Formalin is $10 \%$ formaldehyde solution.

${ }^{5}$ Histological slices: formalin submersion, alcohol submersion, paraffin fixation, microtome cut, fixation in glass and staining.
} 
used for histology, and submerged in formalin. The two slices, guide and sample, are adjacent, so that the histology provides a map of the sample. The colour marks were present in both guide and sample slices, which helped orienting them correctly.

After the SAXS measurement, the samples (already formalin fixed) were introduced in pathological processing cassettes and prepared for the final histo-pathological examination. In this way, a one-to-one correspondence between the classifications by pathology and SAXS patterns was obtained.

This research work was performed in accordance with the ethical regulations of the hospital.

\subsection{Data acquisition and mapping}

The scattering patterns were recorded with the CCD and corrected to spatial distortions and normalised to correspond to the same sample thickness. One-dimensional scattering curves were extracted after azimuthal integration of the two-dimensional patterns.

Some of these scattering patterns are shown in Fig. 3. The differences in the SAXS patterns are clear enough to easily identify tissues.

In order to compare the histology with the scattering patterns, some of the indicators of collagen degradation obtained from the SAXS patterns were used to construct maps of these indicators. The coordinates of measurement spots were retrieved from the positions of the motors, calibrated to the sample holder frame before the experiment started. The colour marks provided reference for sample position in the holder frame, so that the exact location of the measurement point was known.

The $d$-spacing was one of the indicators used for mapping. In this experiment the samples were formalin fixed, and the fixation process removes water from the samples, which may change the axial period. The position of the fifth order collagen peak, in terms of the scattering vector $s$, was retrieved from the

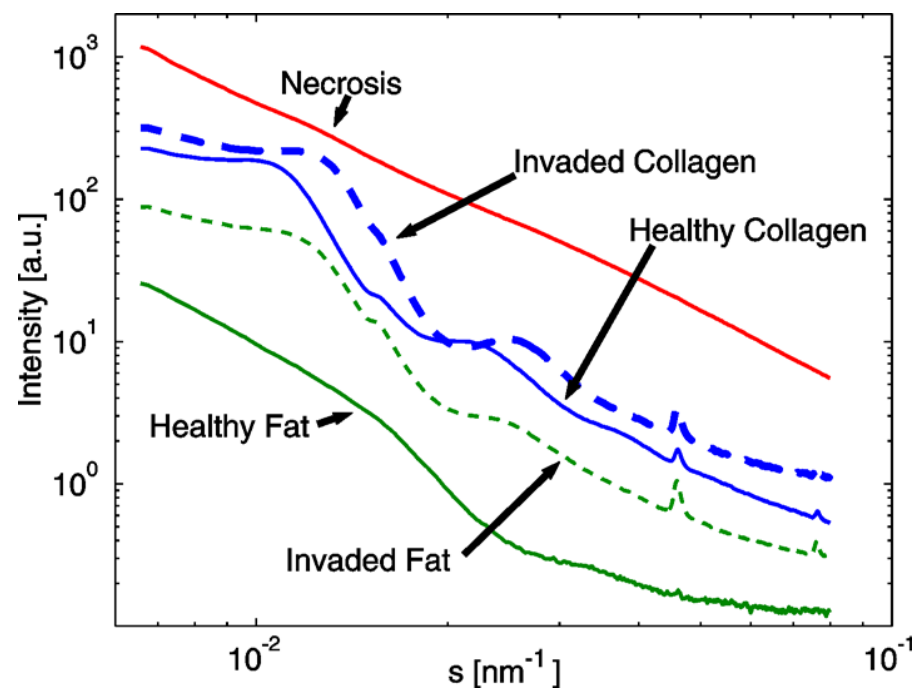

Fig. 3. Tissue characterisation using SAXS patterns. The featureless curve of lowest intensity corresponds to the healthy adipose tissue (fat). Adipose tissue invaded by cancer has diffraction peaks due to the presence of newly formed collagen among the cancer cells (invaded fat). Healthy collagen scattering curve shows the typical collagen peaks, as well as features related to the size and packing of the collagen fibrils. The intensity of scattering from collagen invaded by cancer cells is clearly higher. It preserves, though, the collagen structures in general, but somewhat different. These differences arise from changes in the supra-molecular structure of the collagen. The highest intensity is observed from a necrotic region of a tumour, and the pattern is structureless with a $k^{-2}$ fall-off of intensity, suggesting disintegrated polypeptide coils with a large specific surface. 


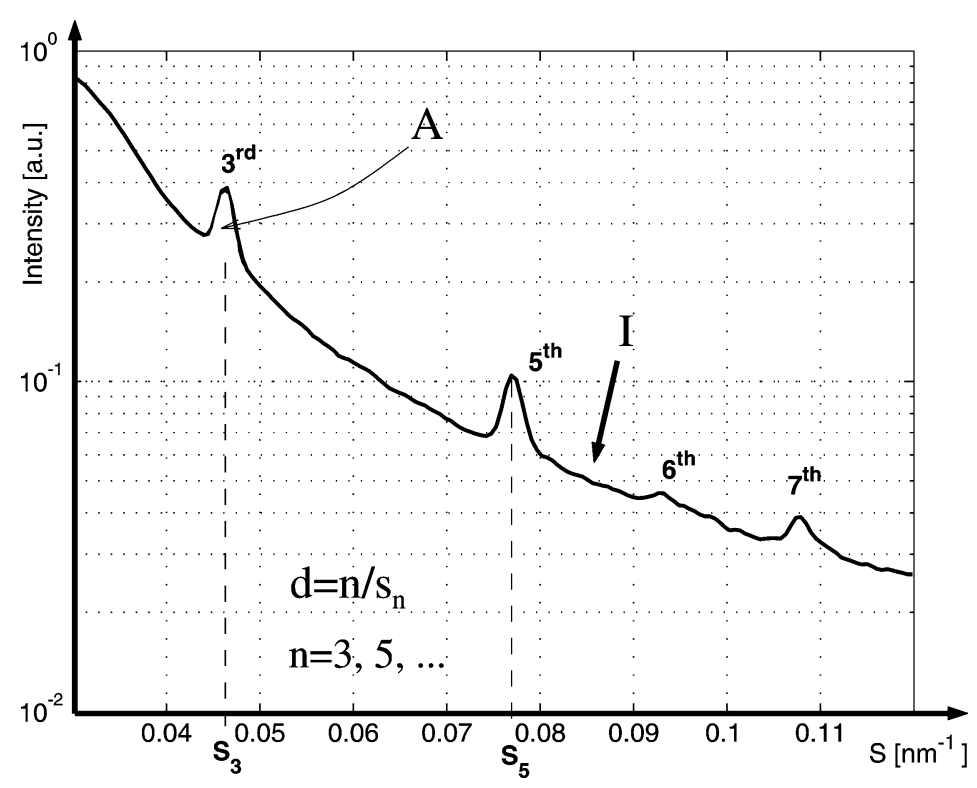

Fig. 4. Schematic scattering pattern of collagen between the 3rd and 7th axial peaks. The area of the third collagen peak $(A)$, the position of the third and fifth peaks $\left(s_{3}, s_{5}\right)$, and the intensity between the peaks $(I)$ are used as indicators.

scattering patterns of all the measurement spots in every sample (see Fig. 4). From these positions the axial $d$-spacing of the collagen fibrils was determined (cf. Eq. (5)),

$$
d=5 / s \text {. }
$$

In general, the values of $d$ were 0.3 to $0.4 \mathrm{~nm}$ smaller than those measured from fresh samples [4].

The second indicator of collagen degradation is the background intensity between collagen peaks (see Fig. 4). It is seen from Eq. (8) that the intensity increases with the surface area of scatterers per unit of volume, and with an increase of the contrast of electron density. Collagen fibrils may suffer of "peelingoff" when they degrade, which increases their surface per unit volume. An observed effect of degradation is the reduction of the fibril diameter. The average intensity in a certain range of the SAXS pattern is plotted in a selected region of the sample. The range is chosen to be the background between two collagen peaks in the regime where the Porod law applies. The range chosen was between the 5 th and the 6 th collagen peaks (or $s$ in the range $0.08-0.09 \mathrm{~nm}^{-1}$ ). In this case, the lower intensities correspond to the adipose tissue, the medium intensities to the healthy collagen, slightly higher intensities to the invaded collagen and the highest intensities to the necrotic tissues. Using the motors positions as reference and the histology, it is possible to build maps of the scattered intensity along certain regions (cf. Figs 5a and $5 b)$.

The axial period of collagen fibrils was measured from all the SAXS patterns obtained from all the samples used in this work. All of them, but two, were histologically classified as carcinoma, either lobular or ductal. However, depending on the way the specimens were prepared, one can find more or less healthy tissue from the tumor surroundings. For this reason, samples marked as malignant in a general pathological diagnosis might be locally benign and, therefore, they present general characteristics of benign tissues. Two examples of this can be found in samples E/16C and G/61B, Table 2. These samples are both parts of the same tumor, a ductal carcinoma. Their histology shows a majority of benign tissue, 

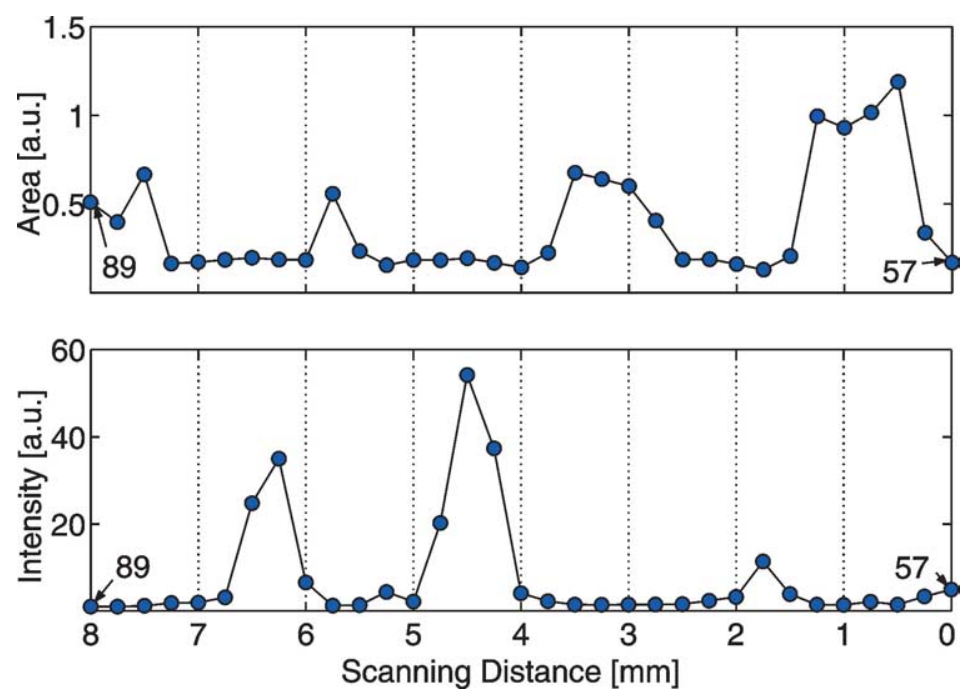

(a)

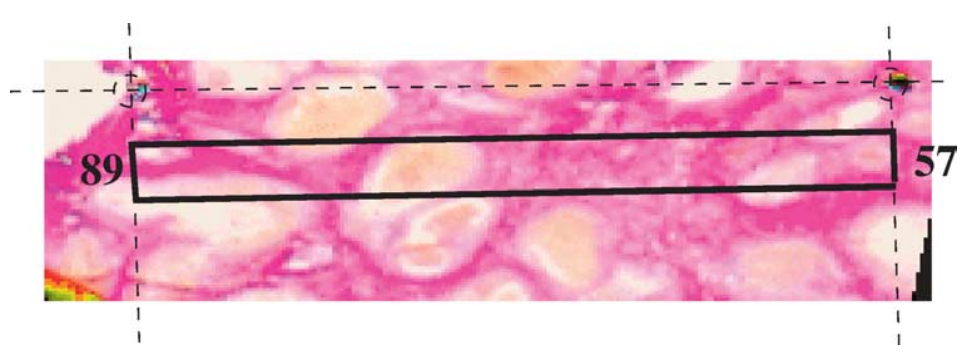

Fig. 5. (a) Mapping of a sample containing islands of necrotic tissue encapsulated in collagen (ductal carcinoma). Top panel, the area under the $3 \mathrm{rd}$ collagen peak (cf. Fig. 4). Step size is $0.25 \mathrm{~mm}$. The bottom panel shows the corresponding variation of the intensity of between the 5rd and 6th collagen peaks. Collagen peaks are small or disappear when the background scattered intensity is high, and vice versa. (b): Histology of the sample. The area where the SAXS patterns were recorded is shown by the rectangle, and the scan numbers are indicated (57 to 89). Pink stain indicates collagen, and yellowish stained tissues are necrotic.

with some islands of in situ ${ }^{6}$ carcinoma and sporadic invasive cells. Therefore, the axial period from these samples is about $0.3 \mathrm{~nm}$ shorter than in the rest of the sample.

The samples used for this experiment were formalin fixed. It was already noted that formalin fixation removes water from the tissues, and reduces the axial period of the collagen fibrils. However, the differences in the axial period are systematically the same as in fresh samples: the period is longer in invaded collagen than in the healthy one. In the earlier work with fresh samples [4], the difference in the axial period was $0.3 \mathrm{~nm}$, while in the present case the difference is $0.2 \mathrm{~nm}$ on the average (cf. Tables 1 and 2).

\section{Conclusions}

We can conclude that the SAXS signal is unique for a given tissue and that it is very useful in the characterisation of breast tissues and their pathologies. The supra-molecular structure of the collagen can be described by a few well-defined parameters, which can be retrieved from the SAXS patterns.

\footnotetext{
${ }^{6}$ Carcinoma is called in situ when it has not invaded adjacent tissues.
} 
Table 1

Collagen $d$ spacing measured from fresh tissues [4]

\begin{tabular}{clrcl}
\hline Code & Tumor type & $N$ & $d(\mathrm{~nm})$ & Notes \\
\hline A & Ca lobulare & 174 & 65.57 & Average over a large region \\
B & Ca lobulare & 3 & 65.39 & Invaded adipose tissue \\
C & Ca ductale & 121 & 65.33 & Average over a large region \\
D & Ca lobulare & 1 & 65.26 & Invaded collagen ('Indian file') \\
E & Ca ductale & 27 & 65.24 & Close to necrotic regions \\
F & Ca ductale & 8 & 65.10 & Far from necrotic regions \\
G & Mastopathic & $47+56$ & 65.02 & Large regions of two benign tumors, \\
H & Fibroadenoma & $40+35$ & 64.87 & same patient \\
J & Ca ductale & 124 & 64.87 & Average over a non-invaded region \\
\hline
\end{tabular}

Table 2

Collagen $d$ spacing measured from formalin fixed tissues, averaged for all the points

\begin{tabular}{llrcl}
\hline Code & Tumor type & $N$ & $d(\mathrm{~nm})$ & Notes \\
\hline 03A/A & Ca lobulare G II & 59 & 64.84 & Average over a large region \\
03B/B & Ca lobulare G II & 106 & 64.78 & Average over a large region \\
16A/C & Ca ductale G III & 18 & 64.78 & Average over a large region \\
16B/D & Ca ductale G III & 105 & 64.79 & Average over a large region \\
16C/E & Ca ductale G III & 88 & 64.61 & Hist: mainly healthy, small Ca in situ \\
61A/F & Ca ductale G I & 121 & 64.83 & Average over a large region \\
61B/G & Ca ductale G I & 74 & 64.58 & Hist: mainly healthy \\
\hline
\end{tabular}

There are differences in these parameters between malignant and benign tumours or healthy tissue, and though small, these differences allow tissue classification. The differences in the collagen structure correlate closely with the histopathology of the tissues at the spatial resolution of this work $(200 \mu \mathrm{m}$ beam at $0.25 \mathrm{~mm}$ scan step).

The formalin fixation is found to change the absolute $d$-spacing of the collagen fibrils, but it does not interfere in the relative differences between healthy and invaded tissues. This is of great value, since the transport and conservation of samples is greatly facilitated by formalin fixation.

Automatic determination of the axial collagen period is difficult, because the differences between different tissues are small. On the other hand, there are large differences in the background intensity and area of the collagen peaks between different tissues, as seen Fig. 3. The average intensity may rise by a factor 5 from healthy to invaded fat, and by more than a factor 10 from healthy collagen to necrotic tissues. These differences can be used for tissue mapping, as shown in Fig. 5. Much systematic work is needed to compare maps of the characteristic parameters of SAXS patterns with histo-pathology of the samples. It is expected that such comparisons provide new information on changes of tissue structures on the molecular level during cancer growth.

\section{Acknowledgements}

The help of the ID02 staff at the ESRF, V. Urban and P. Panini, and advise and discussions with colleagues at ESRF's ID17 and University of Helsinki, A. Bravin, W. Thomlinson, R. Serimaa, M. Torkkeli, 
M. Tenhunen and P. Tuomi, are greatfully acknowledged. The work was funded by the Academy of Finland, project no. 43959, and supported by the ESRF.

\section{References}

[1] A. Bravin, S. Fiedler, P. Coan, J.-C. Labiche, C. Ponchut, A. Peterzol and W. Thomlinson, Comparison between a position sensitive germanium detector and a taper optics CCD "FRELON" camera for diffraction enhanced imaging, Nucl. Instrum. Methods Phys. Res. A $\mathbf{5 1 0}$ (2003), 35-40.

[2] E.F. Eikenberry, B. Brodsky and D.A.D. Parry, Collagen fibril morphology in developing chick metatarsal tendons: 1. X-ray diffraction studies, Int. J. Biol. Macromol. 4 (1982), 322-328.

[3] E.F. Eikenberry, B.B. Brodsky, A.S. Craig and D.A.D. Parry, Collagen fibril morphology in developing chick metatarsal tendon: 2. Electron microscope studies, Int. J. Biol. Macromol. 4 (1982), 393-398.

[4] M. Fernández, J. Keyriläinen, R. Serimaa, M. Torkkeli, M.-L. Karjalainen-Lindsberg, M. Tenhunen, W. Thomlinson, V. Urban and P. Suortti, Small-angle X-ray scattering studies of human breast tissue samples, Phys. Med. Biol. 47 (2002), 577-592.

[5] E.J. Feuer, L.M. Wun, C.C. Boring, W.D. Flanders, M.J. Timmel and T. Tong, The lifetime risk of developing breast cancer, J. Natl. Cancer Inst. 85 (1993), 892-897.

[6] O. Glatter, Small-Angle Scattering International Tables for Crystallography, A.J.C. Wilson and E. Prince, eds, Vol. C, Kluwer, Amsterdam, 2002, Ch. 2.6.

[7] K.E. Kadler, D.F. Holmes, J.A. Trotter and J.A. Chapman, Collagen fibril formation, Biochem. J. 316 (1996), 1-11.

[8] S. Kauppila, Type I and type III collagen synthesis and maturation in malignant tumours, Acta Universitatis Ouluensis, Series D 451, Medica (1998).

[9] J.S. Michaelson, M. Silverstein, J. Wyatt, G. Weber, R. Moore, E. Halpern, D.B. Kopans and K. Hughes, Predicting the survival of patients with breast carcinoma using tumor size, Cancer 95 (2002), 713-723.

[10] I. Pucci-Minafra, C. Luparello, M. Andriolo, L. Basiricò, A. Aquino and S. Minafra, A new form of tumor and fetal collagen that binds laminin, Biochemistry 32 (1993), 7421-7427.

[11] I. Pucci-Minafra, M. Andriolo, L. Basiricò, R. Alessandro, C. Luparello, C. Buccelato, R. Carbelli and S. Minafra, Absence of regular $\alpha_{2}$ (I) collagen chains in colon carcinoma biopsy fragments, Carcinogenesis 19(4) (1998), 575-584.

[12] R. Souhami and J. Tobias, Cancer and Its Management, Blackwell Science Ltd, London, 1998.

[13] P. Suortti and W. Thomlinson, Medical Applications of synchrotron radiation, Phys. Med. Biol. 48 (2003), R1-R35.

[14] T.J. Wess, A.P. Hammersley, L. Wess and A. Miller, A consensus model for molecular packing of type I collagen, J. Structural Biology 122 (1998), 92-100. 


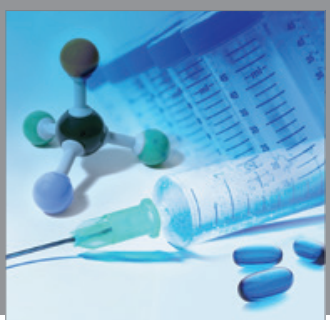

International Journal of

Medicinal Chemistry

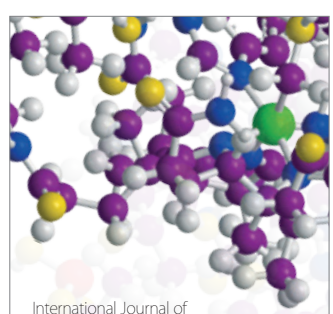

Carbohydrate Chemistry

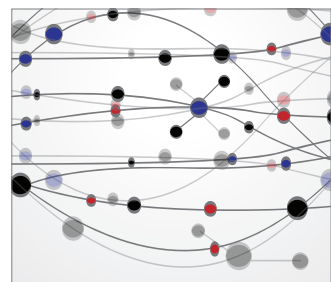

The Scientific World Journal
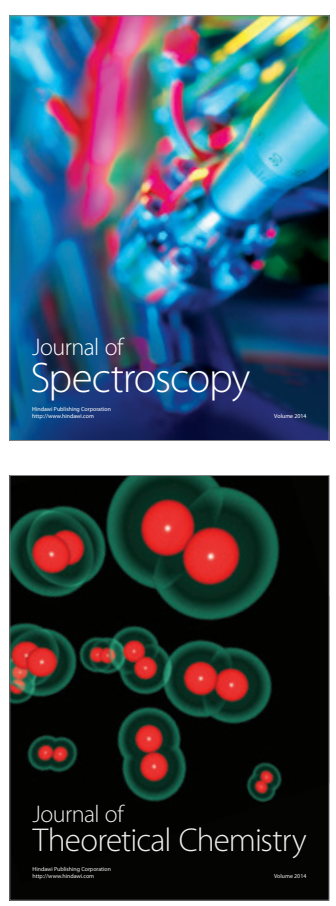
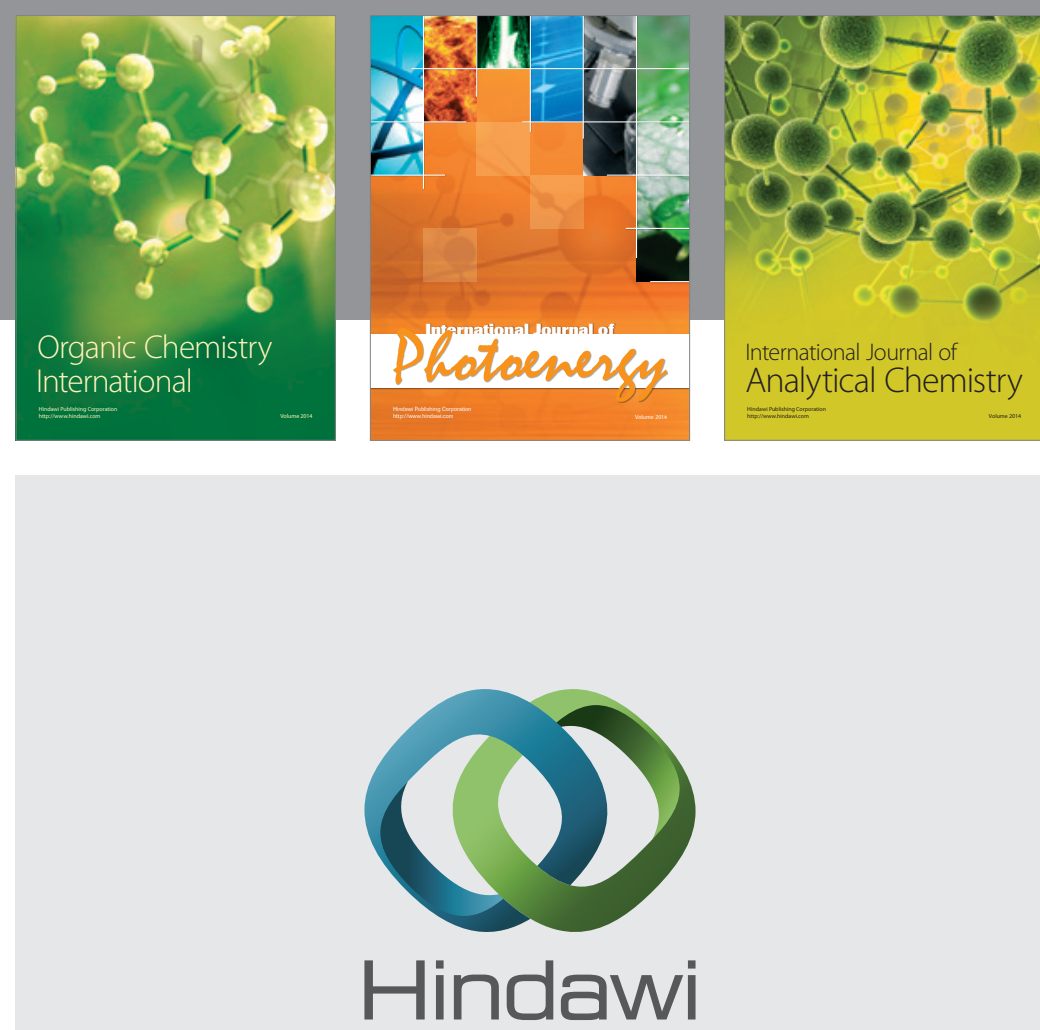

Submit your manuscripts at

http://www.hindawi.com
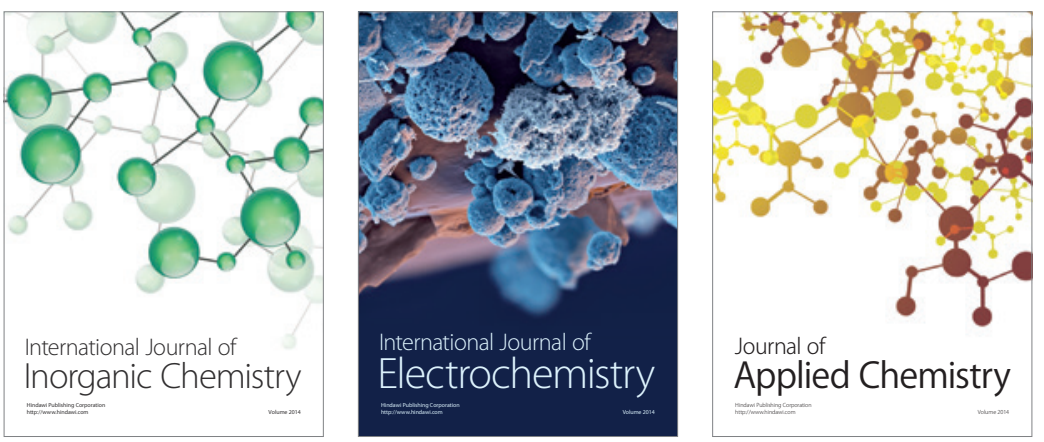

Journal of

Applied Chemistry
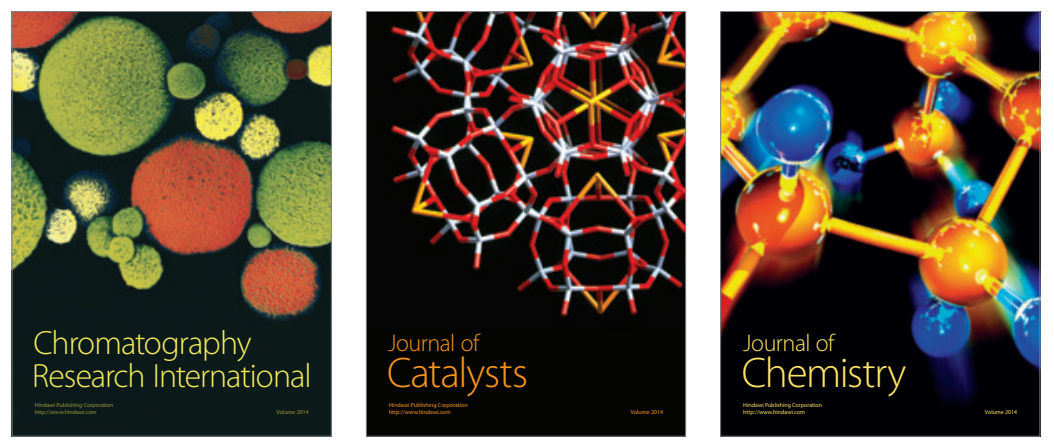
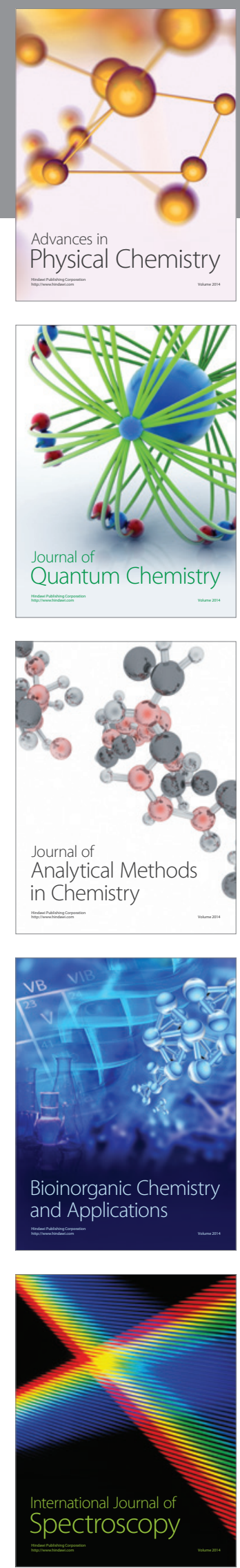